\title{
Spin-pumping and inelastic electron tunneling spectroscopy in topological insulators
}

\author{
Aaron Hurley, Awadhesh Narayan, and Stefano Sanvito \\ School of Physics and CRANN, Trinity College, Dublin 2, Ireland \\ (Received 7 September 2012; revised manuscript received 10 April 2013; published 6 June 2013)
}

\begin{abstract}
We demonstrate that a quantum spin Hall current, spontaneously generated at the edge of a two-dimensional topological insulator, acts as a source of spin pumping for a magnetic impurity with uniaxial anisotropy. One can then manipulate the impurity spin direction by means of an electrical current without using either magnetic electrodes or an external magnetic field. Furthermore we show that the unique properties of the quantum spin Hall topological state have profound effects on the inelastic electron tunneling spectrum of the impurity. For low current densities inelastic spin-flip events do not contribute to the conductance. As a consequence the conductance steps, normally appearing at voltages corresponding to the spin excitations, are completely suppressed. In contrast an intense current leads to spin pumping and generates a transverse component of the impurity spin. This breaks the topological phase yielding to the conductance steps.
\end{abstract}

DOI: 10.1103/PhysRevB.87.245410

PACS number(s): 72.25.Pn, 73.20.-r, 73.40.Gk, 85.75.-d

\section{INTRODUCTION}

The quantum spin Hall (QSH) state is a new topological phase of matter, which has attracted growing attention over the past few years. It is time-reversal invariant and characterized by both a bulk gap and gapless helical edge states with opposite spins counterpropagating at a given edge. In a pathfinding work by Kane and $\mathrm{Mele}^{1}$ it was shown that at low energy a QSH effect can be generated in a sheet of graphene subject to spin-orbit interaction. A topological invariant, $Z_{2}$, distinguishes such a QSH insulator from an ordinary one. ${ }^{2}$ Unfortunately, spin-orbit interaction in graphene is too small to practically realize the QSH state at a realistic temperature; however, a few other options are available. Bernevig et al. predicted that $\mathrm{HgTe} / \mathrm{CdTe}$ quantum wells might exhibit this novel phase, ${ }^{3}$ a prediction soon confirmed by experiments. ${ }^{4,5}$ Three-dimensional analogs of the QSH state have also been found and are generically termed topological insulators (TIs). More recently, evidence for helical edge modes in InAs/GaSb quantum wells has also been found. ${ }^{6}$ Interestingly, both silicene and two-dimensional Ge have been predicted to exhibit the QSH state at experimentally accessible temperatures, ${ }^{7,8}$ and there are theoretical proposals to realize the same in graphene by using nonmagnetic adatoms. ${ }^{9}$ These last two options would yield a material with a low-energy electronic structure identical to that proposed by Kane and Mele.

Given the peculiar spin structure of the QSH state it becomes natural to ask ourselves whether this can be used to manipulate magnetic objects. ${ }^{10}$ In particular, the question we address in this paper is whether spin pumping at the single spin level can be achieved without using spin-polarized electrodes or an external magnetic field. In a nutshell we wish to propose an analog to the numerous recent investigations concerning spin-flip inelastic electron tunneling spectroscopy (SF-IETS) for magnetic adatoms on insulating surfaces, ${ }^{11}$ either in equilibrium or in spin-pumping conditions. ${ }^{12}$

Here we demonstrate that a magnetic impurity deposited at the edge of a $Z_{2}$ TI and presenting a uniaxial magnetic anisotropy, which makes it non-Kondo active, ${ }^{13}$ can be manipulated by the QSH current. Furthermore we show that the topological nature of the QSH state has profound consequences on the SF-IETS conductance spectrum. At low current intensity there is a complete suppression of the conductance steps appearing at the critical biases characteristic of the activation of an inelastic spin-scattering channel. ${ }^{11}$ In contrast, for currents large enough to produce spin pumping the spin of the magnetic impurity is driven away from the anisotropy axis. This breaks the topological protection of the helical edge states and the conductance steps reappear. Intriguingly, in this situation there is a strong dependence of the SF-IETS conductance spectrum on the bias polarity. Our calculations are conducted by using the nonequilibrium Green's function method for transport combined with a perturbative approach to spin scattering from magnetic impurities. ${ }^{14-16}$

\section{MODEL AND COMPUTATIONAL METHODS}

The device that we have in mind is schematically presented in Fig. 1 and consists of two semi-infinite current/voltage electrodes sandwiching a $Z_{2}$ TI ribbon in which a magnetic impurity is absorbed at one of the two edges. Our working hypothesis is that one can construct such a device with either a strong or a weak electronic coupling between the electrodes and the ribbon, i.e., that the interface resistance can be engineered. The entire system is described at the tight-binding level and for the electrodes we use a simple square lattice with hopping parameter $t_{\eta}(\eta=L, R)$.

The TI ribbon has a honeycomb lattice structure with zigzag edge geometry and it is described by the two-dimensional Kane-Mele (KM) Hamiltonian, $H_{\mathrm{KM}}$, which reads

$$
\begin{aligned}
H_{\mathrm{KM}}= & \varepsilon_{0} \sum_{i \alpha} \xi_{i} c_{i \alpha}^{\dagger} c_{i \alpha}+t_{1} \sum_{\langle i j\rangle, \alpha} c_{i \alpha}^{\dagger} c_{j \alpha} \\
& +i t_{2} \sum_{\langle\langle i j\rangle\rangle, \alpha \beta} v_{i j} c_{i \alpha}^{\dagger}\left[\sigma^{z}\right]_{\alpha \beta} c_{j \alpha} .
\end{aligned}
$$

The first term describes a staggered sublattice potential with on-site energy $\varepsilon_{0}$ and $\xi_{i}$ being $\xi_{i}=+1$ for the $A$ sublattice and $\xi_{i}=-1$ for the $B$ one. Here $c_{i \alpha}^{\dagger}\left(c_{i \alpha}\right)$ creates (annihilates) an electron at site $i$ with spin $\alpha$. The second term is the nearest-neighbor hopping with strength $t_{1}$ ( $t_{1}$ sets the energy scale of the problem). Finally the third term, which drives the topological phase, is a second-nearest-neighbor hopping with strength $t_{2}(i=\sqrt{-1})$. This describes the coupling of the 


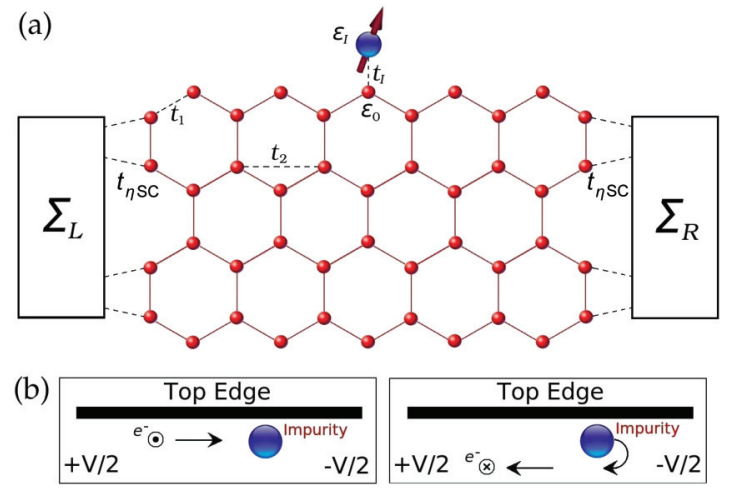

FIG. 1. (Color online) Schematic representation of the device considered in this work (a), comprising a TI with honeycomb lattice structure and a magnetic impurity adsorbed at one of the two edges. In the transport calculations the electronic structures of the electrodes are described by the self-energies, $\Sigma_{\eta}(\eta=L, R)$. In panel (b) the cartoons describe a spin-flip scattering event. A right-going electron with up-spin direction (left panel) is inelastically backscattered by the magnetic impurity. In the process both the electron and the impurity spins are reversed (right panel). Note that, given the topological nature of the ribbon, spin flip forbids electron transmission as the edge presenting a right-going spin-up state does not possess a right-going spin-down one.

electrons orbital motion to their spins via the $z$ component of the Pauli matrices $\left(\sigma^{z}\right)$. The parameter $v_{i j}$ is +1 for counterclockwise hopping and -1 for clockwise.

If we now attach an impurity at site $I$, the total electronic Hamiltonian will become

$$
H_{\mathrm{el}}=H_{\mathrm{KM}}+\varepsilon_{I} \sum_{\alpha} c_{I \alpha}^{\dagger} c_{I \alpha}+t_{I} \sum_{\langle I i\rangle, \alpha} c_{I \alpha}^{\dagger} c_{i \alpha},
$$

where in addition to $H_{\mathrm{KM}}$ one has the on-site potential of the impurity, $\varepsilon_{I}$, and the hopping between the impurity site $I$ and its neighbor $i$ on the honeycomb lattice (with strength $\left.t_{I}\right)$. Finally there are two other terms related to the magnetic impurity spin, $\mathbf{S}$ :

$$
H_{\mathrm{sp}}=D S_{z}^{2} ; \quad H_{\mathrm{el}-\mathrm{sp}}=J_{\mathrm{sd}} \sum_{\alpha \beta} c_{I \alpha}^{\dagger}[\sigma]_{\alpha \beta} c_{I \beta} \cdot \mathbf{S} .
$$

The first, $H_{\mathrm{sp}}$, describes the uniaxial anisotropy (along $z$ ) with $D$ being the zero-field splitting parameter. The second, $H_{\text {el-sp }}$, couples the current-carrying electrons to the local impurity spin with interaction strength $J_{\mathrm{sd}}(\sigma$ is a vector of Pauli matrices). This is usually known as the $s-d$ model for magnetism. ${ }^{17}$

Electron transport is investigated within the LandauerBüttiker approach ${ }^{18}$ implemented by the nonequilibrium Green's function method. ${ }^{19}$ The central quantity to evaluate is the retarded electronic Green's function for the scattering region (the TI ribbon) in the presence of the electrodes, $G^{r}=$ $\left[\left(E+i 0^{+}\right) I-H_{\mathrm{el}}-\Sigma_{\mathrm{L}}-\Sigma_{\mathrm{R}}\right]^{-1}$, where $\Sigma_{\eta}(\eta=\mathrm{L}, \mathrm{R})$ are the electrodes self-energy, which can be computed with standard techniques. ${ }^{20}$ These depend on the hopping parameter between the ribbon and the electrodes, $t_{\eta \mathrm{SC}}$, whose magnitude sets the intensity of the current.

When the conducting electrons couple to the impurity spin $\left(J_{\text {sd }} \neq 0\right)$, the problem becomes intrinsically many body in nature. This is made treatable by constructing a perturbation theory in the electron-spin Hamiltonian, which allows us to incorporate the effect of the electron-spin interaction in an additional self-energy, $\Sigma_{\text {int }}$. In this work we truncate the perturbation expansion to the second order ${ }^{14,16}$ in both the electron and the impurity spin propagator. The latter contains information about the state of the magnetic impurity spins, through the population, $P_{n}$, of the eigenvectors of the spin Hamiltonian, $H_{\text {sp }}$. In particular it is possible to show that the $P_{n}$ 's satisfy a master equation of the form

$$
\frac{d P_{n}}{d t}=\sum_{l}\left[P_{n}\left(1-P_{l}\right) W_{l n}-P_{l}\left(1-P_{n}\right) W_{n l}\right]+\left(P_{n}^{0}-P_{n}\right) / \beta,
$$

where the detailed expression for the transition rates, $W_{l n}$, can be found in Ref. 16, and $\beta=k_{\mathrm{B}} T$ with $k_{\mathrm{B}}$ being the Boltzmann constant and $T$ the temperature. In Eq. (4) the populations $P_{n}^{0}$ are those of the ground state. With this at hand we can compute the current, $I$, and hence by numerical derivative the conductance, $G=d I / d V$.

\section{RESULTS AND DISCUSSION}

We start our discussion by first looking at the transport properties of the ribbon in absence of the magnetic impurity. The relevant quantity here is the spin-resolved total transmission coefficient along a particular edge, ${ }^{21}$ which is given by

$$
T_{\alpha \alpha^{\prime}}^{s}\left(\varepsilon_{\mathrm{F}}\right)=\operatorname{Tr}_{n_{x}}\left[\Gamma_{\mathrm{L}} G^{r} \Gamma_{\mathrm{R}} G^{a}\right]_{\alpha \alpha^{\prime}}^{s},
$$

where $\alpha$ is the spin index $(\alpha=\uparrow, \downarrow), s$ labels the edges $(s=$ top, bottom) and $G^{a}$ is the advanced Green's function. The trace is over the number of atoms, $n_{x}$, along the given edge and the transmission coefficient is evaluated at the Fermi energy, $\varepsilon_{\mathrm{F}}$. As a matter of notation a $\left(n_{x}, n_{y}\right)$ ribbon contains $n_{x}$ atoms in the direction of transport and $n_{y}$ along the transverse one. When the Fermi level is fixed at the half-filling point the ribbon is insulating in the bulk, but presents edge topological protected states (here $t_{\eta}=t_{1}=1$, and $t_{2}=t_{1} / 3$, which ensures that the KM Hamiltonian describes a QSH state). In this situation we find for an $(11,6)$ ribbon, $T_{\uparrow \uparrow}^{\text {top }}=0.9$, $T_{\downarrow \downarrow}^{\text {top }}=0.1, T_{\uparrow \uparrow}^{\text {bottom }}=0.1$, and $T_{\downarrow \downarrow}^{\text {bottom }}=0.9$. Such values demonstrate that the current along the QSH edges is spin polarized, although not completely because of the finite size of the ribbon. Calculations for a $(7,4)$ ribbon give us $T_{\uparrow \uparrow}^{\text {top }}=0.85$, $T_{\downarrow \downarrow}^{\text {top }}=0.15, T_{\uparrow \uparrow}^{\text {bottom }}=0.15$, and $T_{\downarrow \downarrow}^{\text {bottom }}=0.85$.

We now switch on the magnetic interaction between an $S=1$ local spin and an $(11,6)$ ribbon. In general we place the impurity at the center of the edge and choose a coupling parameter $t_{I}$ and an on-site energy $\varepsilon_{I}$ to ensure that the density of states localized at the impurity site $\rho_{I}(E)$ is approximately constant for energies $E$ around the Fermi level (this ensures the convergence of the perturbation scheme ${ }^{14}$ ). The exchange coupling $J_{\mathrm{sd}}$ is chosen so that the perturbation parameter $\rho_{I} J_{\text {sd }}$ is approximately 0.1 . These conditions are satisfied for $\varepsilon_{I}=J_{\text {sd }}=t_{1} / 2$ and $t_{I}=t_{1} / 4$. The spin degeneracy is lifted by an axial anisotropy $D=-10^{-3} t_{1}$, which corresponds to $D=-2.0 \mathrm{meV}$, if the nearest-neighbor hopping in the ribbon is fixed at a reasonable value of $t_{1}=2 \mathrm{eV}\left(k_{\mathrm{B}} T=0.05\right)$. 
The uniaxial anisotropy gives us a degenerate ground state with the two spin states $|-1\rangle$ and $|+1\rangle$ separated from the first excited state $|0\rangle$ by $|D|$. As a result we do not expect a Kondo-like behavior since no allowed transition between the degenerate ground state may occur. The second order perturbation expansion is then well justified. The values $t_{\eta}=$ $4 t_{\eta \mathrm{SC}}=t_{1}$ ensure that the spin system remains in equilibrium, i.e., in its ground state, throughout the spin-scattering process.

Figure 2 shows the calculated conductance spectra, $G(V)$, normalized to the $V=0$ conductance, $G_{\text {el }}$, for three values of the parameter governing the QSH state, $t_{2}$. For $t_{2}=0$ there are no topologically protected edges and we observe the characteristic inelastic conductance step at a voltage $V=D / e$, when the transition from the ground state to $|0\rangle$ becomes possible ( $e$ is the electron charge). However, as $t_{2}$ is increased and we enter into the topological phase, we reveal a suppression of the inelastic contribution to the conductance, with an almost full suppression at the maximum value of $t_{2}=t_{1} / 3$. The cartoon in Fig. 1(b) helps one to understand the mechanism for such a suppression. At a positive bias, the right-going current is up-spin polarized. This means that the $|-1\rangle \rightarrow|0\rangle$ transition scatters out spin-down electrons. These cannot propagate towards the right electrode since there is no right-moving spin-down state in the upper edge and, as a consequence, they are completely reflected. Hence, as spin-flip events can only lead to backscattered electrons, the inelastic channel does not contribute to the current. Note that the residual conductance increase in Fig. 2 for $t_{2}=t_{1} / 3$ is simply due to the finite size of the ribbon.

We now investigate the possibility of manipulating the impurity spin direction. This is achieved by increasing the overall conductance, i.e., by increasing the average current density. When one works with a scanning tunneling microscopy setup bringing the tip closer to the impurity ${ }^{12}$ does the job, while here our control parameter is the electronic coupling between the leads and the ribbon, $t_{\eta \mathrm{SC}}$. As such all the calculations that follow have been performed with $t_{\eta \mathrm{SC}}=t_{1}$.

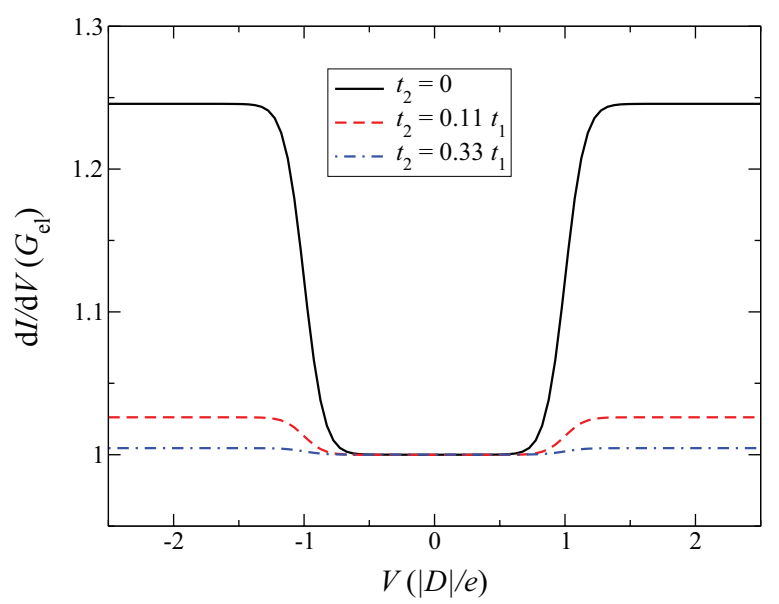

FIG. 2. (Color online) Spin-polarized IETS conductance spectrum for a TI $(11,6)$ ribbon with an $S=1$ magnetic impurity attached at the upper edge. Note that the conductance step at the voltage characteristic of the inelastic excitation gets suppressed as the $t_{2}$ parameter is increased, i.e., as the ribbon is brought well inside the topological region of the phase diagram.
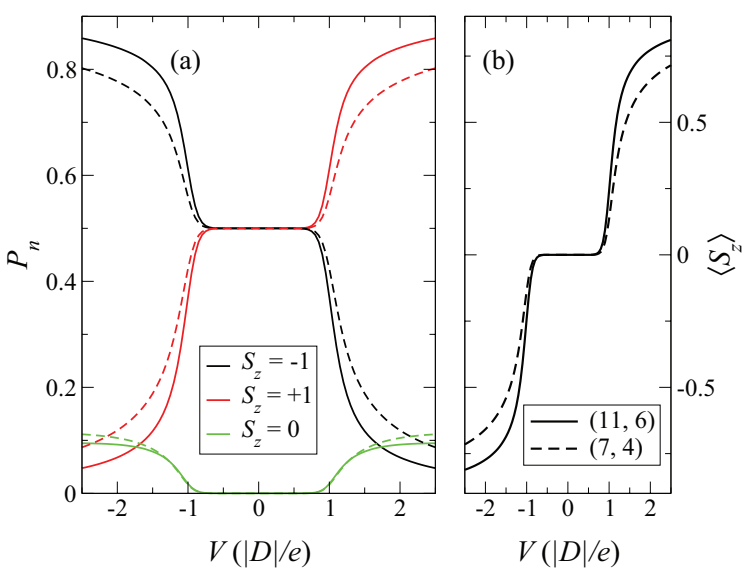

FIG. 3. (Color online) (a) Nonequilibrium population as a function of bias of the $S=1$ impurity spin states for a $(7,4)$ (broken lines) and an $(11,6)$ ribbon (solid lines). In panel (b) we show the average magnetization of the impurity for the same ribbons.

The calculated populations of the various spin states are plotted as a function of bias in Fig. 3(a) for both an $(11,6)$ and a $(7,4)$ ribbon. An $S=1$ spin in a uniaxial anisotropy field and in thermal equilibrium with an electron bath presents an equal probability to occupy the $|+1\rangle$ and the $|-1\rangle$ states, i.e., for $V=0$ one has $P_{+1}=P_{-1}=1 / 2$. As soon as the bias is increased at and above $|D| / e$, excitations to the $|0\rangle$ state become possible due to spin-flip backscattering. In this case, however, the current is intense, so that in between two scattering events the impurity spin does not have the time to relax back to the degenerate ground state. This means that now a spin-up electron (the majority specie in the upper edge right-going channel) can also induce the transition $|0\rangle \rightarrow|+1\rangle$. The consequence is that the electronic current flowing at the upper edge, in virtue of its spin polarization and its intensity, produces a net flow of population between the two degenerate ground states, i.e., for $V>+|D| / e$ one has $P_{+1}>P_{-1}$. In other words the impurity spin is driven by the current away from its uniaxial anisotropy axis. This can be fully appreciated by looking at Fig. 3(b), where we show the average magnetization $\left\langle S^{z}\right\rangle=\sum_{m} P_{m} S_{m}^{z}$ as a function of bias. Such spin pumping is essentially identical to what happens for spin-polarized tips ${ }^{22}$ except that now one does not need either a magnetic electrode or an external magnetic field. Note that at a negative bias the effect is reversed, i.e., for $V<-|D| / e$ one has $P_{-1}>P_{+1}$, and that placing the impurity on the lower edge is equivalent to reversing the bias polarity.

The effects of the spin pumping on the shape of the conductance spectrum are finally presented in Fig. 4. This time the $G(V)$ trace presents a step at the voltage corresponding to the $| \pm 1\rangle \rightarrow|0\rangle$ transition, i.e., the electron transport becomes sensitive to spin-flipping events. Such an appearance of the conductance step signals the suppression of the topological helical states induced by the transverse magnetization of the spin impurity. ${ }^{23}$ Intriguingly, the magnitude and sign of the conductance step depends on the bias polarity. In particular we note that there is an inelastic contribution, which is symmetric with respect to the sign of $V$, and always contributes to enhance the conductivity. In contrast the elastic contribution is antisymmetric with respect to the bias polarity, i.e., the 


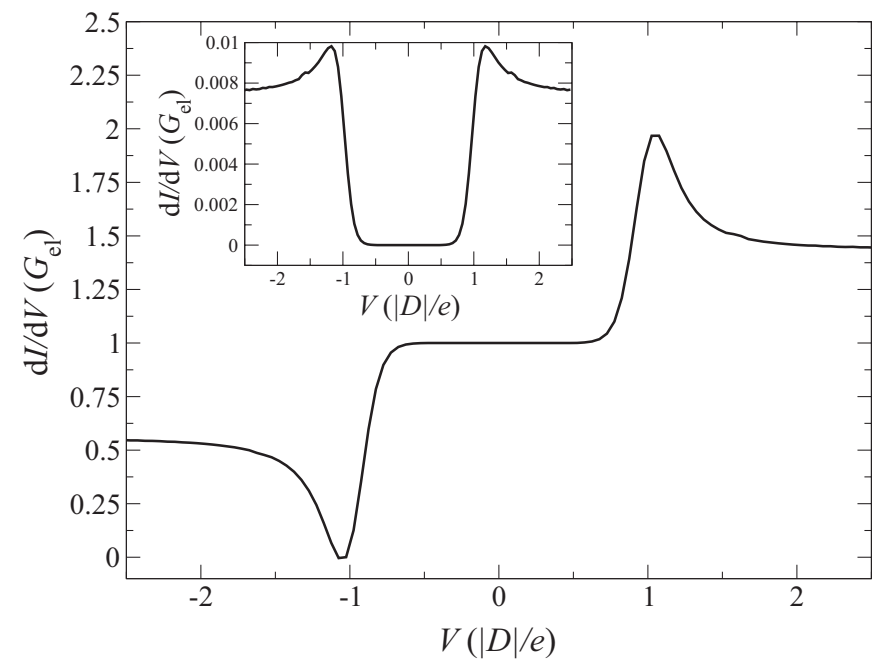

FIG. 4. Spin-polarized IETS conductance spectrum for a TI $(11,6)$ ribbon with an $S=1$ magnetic impurity attached at the upper edge. In this case the current is intense and drives the impurity spin away from the uniaxial anisotropy axis (see Fig. 3). Notably now there is a step in the differential conductance at the voltage corresponding to the inelastic transition $| \pm 1\rangle \rightarrow|0\rangle$. The magnitude and sign of such step depends on the bias polarity. The inset shows the inelastic contribution to the conductance.

elastic current increases for $V>|D| / e$ and decreases for $V<-|D| / e$. Placing the impurity on the opposite edge yields a mirror symmetric situation. This time the magnetization is driven toward $\left\langle S_{z}\right\rangle=-1\left(\left\langle S_{z}\right\rangle=+1\right)$ and the conductance decreases (increases) for a positive (negative) bias voltage. Overall we can summarize our results by noting that the sign of the change in the conductance trace at the onset voltage $|V|=|D| / e$ is proportional to $(\mathbf{v} \times \sigma) \cdot\langle\mathbf{S}\rangle$, where $\mathbf{v}$ is the group velocity of the edge state. Thus, the antisymmetry of the conductance is related to the helicity of the edge state, $\mathbf{v} \times \sigma$.

When one looks at the perturbative expansion of the conductance it can be realized that the term giving rise to the bias asymmetry is the magnetoresistive elastic term of the $s-d$ Hamiltonian. Its contribution to the self-energy reads

$\left[\Sigma_{\text {mag-elas }}^{\gtrless}(E)\right]^{(2)} \propto-J_{s d}^{2} \sum_{m n} G_{0}^{\gtrless}\left(E \pm \Omega_{m n}\right) \delta_{m n} P_{n} S_{m n}^{z}$,

where $G_{0}^{\gtrless}$ is the electronic Green's function, $\Omega_{m n}$ is the energy difference between the two spin states $|n\rangle$ and $|m\rangle$, and $S_{m n}=$ $\left\langle m\left|S_{z}\right| n\right\rangle$ is the spin transition matrix elements. Since the term includes $\delta_{m n}$ there is only an elastic contribution $\left(\Omega_{n n}=0\right)$, which involves no spin-flip events. ${ }^{16} \mathrm{Such}$ term is proportional to $S_{n n}^{z}$ and thus reverts its sign as the direction of impurity spin is reversed. Note that the elastic and inelastic contributions to the conductance are calculated by partitioning the current into two parts, obtained respectively from the elastic and inelastic energy-dependent self-energies. These, however, are evaluated from the same self-consistent electronic Green's function, meaning that the elastic and inelastic contributions are not completely disentangled. As such, it should not be surprising that the onset of inelastic scattering is evident also in the elastic contribution to the conductance.

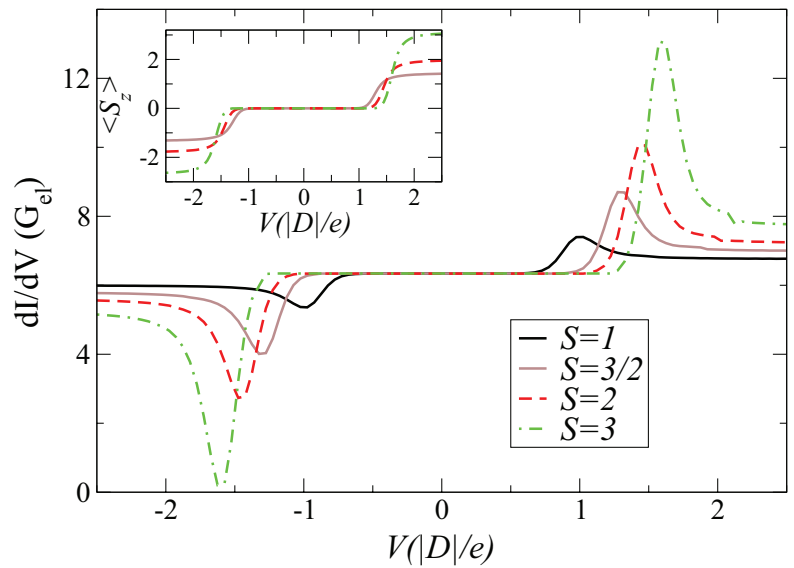

FIG. 5. (Color online) Spin-polarized IETS conductance spectrum for a TI $(11,6)$ ribbon incorporating a magnetic impurity with various $\operatorname{spin}(S=1,3 / 2,2,3)$ attached at the upper edge, in the intense current regime. The step in the differential conductance increases in magnitude with increasing the spin value of the adatom. Note that the spectra have been aligned vertically for clarity of comparison. The inset shows the average magnetization of the impurity for different values of $S$. Note that spin pumping persists for the larger values of the impurity spin.

Finally we investigate how the conductance profile changes as we increase the value $S$ of the total spin of the magnetic impurity. This is done by rescaling the anisotropy parameter $D$ and the electron-spin coupling strength $J_{\text {sd }}$ respectively to $D /|S|$ and $J_{\text {sd }} /|S|$, so that the effective interaction strength and the total spin anisotropy do not change in the comparison. Our results are plotted in Fig. 5. The figure reveals that, as the total spin increases, the height of the differential conductance steps gets larger. In the figure we also plot the average magnetization as a function of bias, which indicates that the spin pumping is present for larger values of $S$ and that for voltages exceeding the energy of the inelastic transition the average value of $S_{z}$ approaches its maximum value.

\section{CONCLUSION}

In conclusion we have demonstrated that a QSH current flowing at the edge of a $Z_{2}$ TI can be used to manipulate the spin of a magnetic impurity. This does not require either an external magnetic field or magnetic electrodes, i.e., it allows one to implement spintronics without magnetism. Importantly the fingerprint of the manipulation can be found in the conductance profiles themselves, making SF-IETS a tool for preparing, manipulating, and reading a quantum spin in the solid state.

\section{ACKNOWLEDGMENTS}

This work is supported by Science Foundation of Ireland (A.H., Grant No. 07/IN.1/I945) and Irish Research Council for Science, Engineering and Technology (A.N.). Computational resources have been provided by Trinity Center for High Performance Computing. We thank Cyrus Hirjibehedin, Magnus Paulsson, and Nadjib Baadji for illuminating discussions. 
${ }^{1}$ C. L. Kane and E. J. Mele, Phys. Rev. Lett. 95, 226801 (2005).

${ }^{2}$ C. L. Kane and E. J. Mele, Phys. Rev. Lett. 95, 146802 (2005).

${ }^{3}$ B. A. Bernevig, T. L. Hughes, and S. C. Zhang, Science 314, 1757 (2006).

${ }^{4}$ M. König, S. Wiedmann, C. Brüne, A. Roth, H. Buhmann, L. W. Molenkamp, X. L. Qi, and S. C. Zhang, Science 318, 766 (2007).

${ }^{5}$ A. Roth, C. Brüne, H. Buhmann, L. W. Molenkamp, J. Maciejko, X. L. Qi, and S. C. Zhang, Science 325, 294 (2009).

${ }^{6}$ I. Knez, R. R. Du, and G. Sullivan, Phys. Rev. Lett. 107, 136603 (2011).

${ }^{7}$ C.-C. Liu, W. Feng, and Y. Yao, Phys. Rev. Lett. 107, 076802 (2011).

${ }^{8}$ M. Ezawa, New J. Phys. 14, 033003 (2012).

${ }^{9}$ C. Weeks, J. Hu, J. Alicea, M. Franz, and R. Wu, Phys. Rev. X 1, 021001 (2011).

${ }^{10}$ A. M. Lunde and G. Platero, Phys. Rev. B 86, 035112 (2012).

${ }^{11}$ A. J. Heinrich, J. A. Gupta, C. P. Lutz, and D. M. Eigler, Science 306, 466 (2004).
${ }^{12}$ S. Loth, C. P. Lutz, and A. J. Heinrich, Nat. Phys. 6, 340 (2010).

${ }^{13}$ J. Maciejko, Phys. Rev. B 85, 245108 (2012).

${ }^{14}$ A. Hurley, N. Baadji, and S. Sanvito, Phys. Rev. B 84, 035427 (2011).

${ }^{15}$ A. Hurley, N. Baadji, and S. Sanvito, Phys. Rev. B 84, 115435 (2011).

${ }^{16}$ A. Hurley, N. Baadji, and S. Sanvito, Phys. Rev. B 86, 125411 (2012).

${ }^{17}$ K. Yosida, Theory of Magnetism (Springer-Verlag, Berlin, 1998).

${ }^{18}$ M. Buttiker, Phys. Rev. B 38, 9375 (1988).

${ }^{19}$ S. Datta, Electronic Transport in Mesoscopic Systems (Cambridge University Press, Cambridge, 1997).

${ }^{20}$ I. Rungger and S. Sanvito, Phys. Rev. B 78, 035407 (2008).

${ }^{21}$ L. Sheng, D. N. Sheng, C. S. Ting, and F. D. M. Haldane, Phys. Rev. Lett. 95, 136602 (2005).

${ }^{22}$ F. Delgado and J. Fernandez-Rossier, Phys. Rev. B 82, 134414 (2010).

${ }^{23}$ A. Narayan and S. Sanvito, Phys. Rev. B 86, 041104(R) (2012). 